\title{
Coulomb Interaction Effect in Quantum Transport
}

\author{
M. Crisan · I. Grosu
}

Received: 19 January 2010 / Accepted: 30 March 2010 / Published online: 20 April 2010

(C) The Author(s) 2010. This article is published with open access at Springerlink.com

\begin{abstract}
We study the quantum Anderson model in the presence of the finite Coulomb interaction $U$ using the $t$-matrix approximation. The energy of the $d$-electron level increases as $U \ln (U / \pi \Delta)$ for strong Coulomb interaction $U$ and finite level-width $\Delta$. In the case of small $U / \Delta$ parameter this approximation gives the results from the perturbation theory. The spin susceptibility is calculated as a function of $U$ and compared with the results obtained by renormalized perturbation approximation. The results can be used to study the effect of the finite Coulomb interaction in the quantum transport.
\end{abstract}

Keywords Anderson model · Quantum transport

\section{Introduction}

The Kondo effect is a well-known example of the strongly correlated system in condensed matter physics [1]. The basic idea of it is the occurrence of a resonance around the Fermi energy, known as the Kondo resonance, due to the screening of the impurity spin by the conduction electrons. Historically, the Kondo effect appeared about forty years ago to explain the minimum for decreasing temperature observed in dilute alloys containing magnetic impurities. According to the microscopic theory, when temperature $T$ decreases below the Kondo temperature $T_{\mathrm{K}}$, the localized magnetic impurity starts to interact strongly with the surrounding electronic cloud, generating a singlet many-body state. The minimum in the resistivity results from the fact that, as the temperature is lowered, the scattering with phonons decreases

M. Crisan $(\varangle) \cdot$ I. Grosu

Department of Theoretical Physics, University of Cluj, 400084

Cluj-Napoca, Romania

e-mail: mircea.crisan@gmail.com down to the Kondo temperature at which the scattering with localized impurities becomes important as the Kondo effect is operative. This is traditional Kondo effect when the magnetic impurities act as scattering centers, increasing the sample resistivity. The opposite behavior is the Kondo effect in the quantum dots (QD) which are small droplets of electrons spatially confined, and which can be modeled by a metallic grain. The analogy between the conduction in the coupled metallic grains and the conduction in quantum dots is very useful in order to point out the importance of the Coulomb interaction effect in the quantum transport from the quantum dots. The parameter which determines physical properties of the granular array is the tunneling conductance between the grains. The important parameter for the calculation of physical properties of these systems is the Coulomb charge energy $E_{\mathrm{c}}$. This energy is equal to the variation in the grain energy when one electron is added or removed from the grain, and gives in fact the charge quantization. The coupling of the single grain to a metallic reservoir gives rise to the Coulomb blockade which appears only if this coupling is weak. The theoretical advances in the study of the transport in the granular systems are extensively presented by Beloborodov et al. [2]. The average charge of the grain as a function of voltage can be measured using QD capacitively coupled to a grain setup which is known as Coulomb staircase [3]. In this case the Coulomb blockade can be modified by the width $\Gamma$ of the resonance level from QD and if $\Gamma \ll E_{\mathrm{c}}$, a perfect transmission is obtained.

These considerations show that even in a simple QD the Coulomb interaction has an important effect which is relevant for the weak coupling between dot and the metallic contacts (leads).

The main difference between the transport in granular systems and the quantum dots systems is due by the absence of the disorder and fluctuation effect, which are not 
considered in the conduction mechanism which is basically determined by the Kondo resonance near the Fermi energy of the leads.

The standard model, in this case, consists of a QD connected to two leads in such a way that electrons transmitted from one electrode to another should pass through the QD, and the theoretical calculation predicted the increasing of the conductance when decreasing temperature, and the Kondo effect sets in due to a resonant transmission through the Kondo resonance which appears in the local density of states at the dot site at the Fermi level. In this case the conductance at $T=0$ takes the limiting value $2 e^{2} / h$.

The single impurity Anderson model (SIAM) has been used extensively to explain the Kondo effect in dilute alloys and after the discovery of the Kondo state in QD it is usually considered in the quantum transport theory. The transport coefficients require accurate expressions for both the temperature and frequency dependence of the impurity Green function, a quantity which is difficult to calculate in the strong correlation limit of the Anderson model. The most efficient method is the Wilson renormalization group method, which is non-perturbative and is therefore accurate for an arbitrary large Coulomb interaction. However, an analytical solution is important for this model in order to get a better understanding of a new problem as quantum transport in quantum dots or the conduction in carbon nanotubes with magnetic impurities. A rapidly convergent perturbation method was first proposed by Yoshida and Yamada [4], Yamada [5] and developed by Horvatić and Zlatić [6] to calculate the density of states of localized electrons for the asymmetric non-degenerate Anderson model. The results from [2] agree with the numerical calculation from [7] and with the phenomenological Fermi liquid theory of Noziéres [8]. It is remarkable that their perturbational expansion converges very rapidly even for the values of $u=U / \pi \Delta$ which are above a non-magnetic boundary.

Using the scaling equations, Haldane [9] calculated the energy of the $d$-level shifted by the mixing $\Delta$ as $E^{*}=E_{d}+$ $(\Delta / \pi) \ln (D / \Delta)$, where $D$ is the bandwidth if $U$ is larger than $D$. In the case $U<D$, Haldane suggested that $D$ has to be of the order of $U$ because the scattering of the conduction electrons with energy higher than $U$ is canceling and the cutoff for the energy is $U$ and the self-energy of the $d$-electrons has an ultraviolet divergence.

The purpose of this paper is to show that even in the $t$-matrix approximation the perturbation theory reproduces this result for large $U$. In the approximation of small $U$ we re-obtain the Yoshida and Yamada [4] results up to the second order terms in the parameter $u$. The rest of the paper is organized as follows. In Sect. 2 we present the model and calculate the self-energy of the $d$-electrons. The $t$-matrix approximation was also used by Schrieffer and Mattis [10] to discuss the influence of the correlation effect on the localized magnetic moments which have been used first in the
Hartee-Fock approximation. This approximation gives correct results, in our calculation, up to the order of $U^{2}$, and this limitation is generated by the ladder approximation used in the calculation of the $t$-matrix.

The density of states is calculated, for the large and small Coulomb interaction, in Sect. 3. In Sect. 4 we calculate the magnetic susceptibility in the same limits. In Sect. 5 we discuss the relevance of these analytical results which are compared with the results obtained by Hewson [9] by renormalized perturbation theory.

The results can be used for the calculation of the influence of the finite Coulomb interaction in the quantum transport, and such an approach has been used by Son et al. [13] in the calculation of the current in an Aharonov-Bohm interferometer.

\section{Model}

We start the model with the Anderson Hamiltonian:

$$
\begin{aligned}
H= & \sum_{\mathbf{k}, \sigma} \epsilon_{k} n_{\mathbf{k}, \sigma}+\sum_{\sigma} E_{d} n_{\sigma}+U n_{d \uparrow} n_{d \downarrow} \\
& +\sum_{\mathbf{k}, \sigma}\left[V_{k d} c_{\mathbf{k}, \sigma}^{\dagger} d_{\sigma}+h . c\right]
\end{aligned}
$$

where $\epsilon_{\mathbf{k}}$ is the energy of the conduction electrons from the leads, $E_{d}$ is the energy of the $d$-level, $U$ is the Coulomb interaction between $d$-electrons and $V_{k d}$ is the interaction between the conduction electrons and the localized $d$-levels. We consider the bandwidth $D$ very large and the dependence of physical quantities of $U$ will be derived in the approximation valid for low electron density, which is the particleparticle ladder used in [10].

The Green function of the $d$-electrons has the general form:

$G_{\sigma}(\omega)=\frac{1}{\omega-E_{d}-\Sigma_{\sigma}(\omega)+i \Delta \operatorname{sgn} \omega}$

where $\Delta=\pi \sum_{\mathbf{k}}\left|V_{k d}\right|^{2} \delta\left(\omega-\epsilon_{\mathbf{k}}\right)$. The self-energy $\Sigma_{\sigma}(\omega)$ is given by:

$\Sigma_{\sigma}(\omega)=\int d z G_{-\sigma}(z-\omega) t(\omega)$

and $t$-matrix is calculated in the low-density approximation as:

$t(\omega)=\frac{U}{1+U \Pi(\omega)}$

where polarization function $\Pi(\omega)$ is given by:

$\Pi(\omega)=\int \frac{d z}{2 \pi i} G_{\uparrow}(z+\omega) G_{\downarrow}(-z)$ 
In order to calculate the self-energy, we transform the expression of polarization function $\Pi(\omega+i 0)$ as:

$$
\begin{aligned}
\Pi(\omega+i 0)= & -\frac{1}{\pi} \int_{-\infty}^{0} d z[G(-z+\omega+i 0) \operatorname{Im} G(z+i 0) \\
& +G(z+\omega+i 0) \operatorname{Im} G(-z-i 0)]
\end{aligned}
$$

and using $\operatorname{Im} G(z-i 0)=-\operatorname{Im} G(z+i 0)$, we get the general relation

$$
\begin{aligned}
& \Pi(\omega+i 0) \\
& =-\frac{1}{\pi}\left[\int_{-\infty}^{0} d z G(-z+\omega+i 0) \operatorname{Im} G(z+i 0)\right. \\
& \left.\quad+\int_{0}^{\infty} d z G(-z+\omega+i 0) \operatorname{Im} G(z+i 0)\right]
\end{aligned}
$$

which is necessary for the calculation of the polarization function (5). In order to obtain an analytical expression for $\Pi(\omega)$, we approximate the energy dependence of the selfenergy by its value at the Fermi energy $\Sigma(0)$. Using this approximation we calculate:

$$
\begin{aligned}
& F(x, y) \\
& \quad=\pi \Delta \Pi(\omega+i 0) \\
& \quad=-\frac{2}{x-2 y}\left[\arctan y+\frac{1}{x-2 y+2 i} \ln \left(1-\frac{i x}{1+i y}\right)\right]
\end{aligned}
$$

where $x=\omega / \Delta$ and $y=\left(\Sigma(0)+E_{d}\right) / \Delta$. Using (3) and (8), we obtain:

$y=-\operatorname{Im} \int_{-\infty}^{0} d x \frac{1}{[1 / u+F(x, y)](x-y+i)}$

In the following we will calculate the self-energy $\Sigma(0)$ for the cases of large $(u \gg 1)$ and small $(u \ll 1)$ Coulomb interactions. Let us consider the case of large $U$. In this case from (8) we approximate the polarization function as:

$F(x, y) \simeq \frac{-2}{x} \arctan y$

which can be given also by the total number of $d$-electrons $n$ using the equation:

$n=\frac{1}{2}-\frac{1}{\pi} \arctan y$

For the case $u \gg 1$ we perform integration from (9) using for $\Pi(x, y)$ the approximation given by (10), and in limit for large $u$ we get:

$y \simeq \frac{1}{\pi} \ln \frac{\pi u}{y}$ an equation which has the solution the Lambert function and can be approximated as:

$E_{d}+\Sigma(0)=E_{d}+\frac{\Delta}{\pi}\left[\ln \left(\frac{U}{\Delta}\right)-\ln \ln \left(\frac{U}{\Delta}\right)\right]$

If $U<D$, the energy of the $d$-level is given by the Haldane expression:

$E_{d}^{*}=E_{d}+\frac{\Delta}{\pi} \ln \left(\frac{U}{\Delta}\right)$

and obtained by scaling equations.

The Green function $G(\omega)$ has the form:

$G_{\sigma}(\omega)=\frac{1}{\omega-E_{d}^{*}+i \Delta \operatorname{sgn} \omega}$

For the case of small $u$ we take the same approximation given in (10) for $\Pi$, which was giving reasonable approximations for large $u$.

The second-order contribution in $U^{2}$ has the form

$\Sigma^{(2)}=U^{2} \frac{1}{(2 \pi)^{2}} \int_{-\infty}^{\infty} d z \int_{-\infty}^{\infty} d \omega G(\omega) G(z) G(\omega-z)$

where $G(z)$ is given by

$G(z)=\frac{1}{z-E_{d}-\Sigma(0)+i \Delta \operatorname{sgn} z}$

In order to calculate this contribution, we approximate $G(z)$ by

$G(z) \simeq \frac{1}{z+i \Delta \operatorname{sgn} z}\left[1+\frac{\left(\Sigma(0)+E_{d}\right)}{z+i \Delta \operatorname{sgn} z}\right]$

Using this approximation, the integral from (16) can be given in terms of the Green function

$G_{0}(z)=\frac{1}{z+i \Delta \operatorname{sgn} z}$

as:

$$
\begin{aligned}
\Sigma^{(2)}= & \frac{U^{2}\left(\Sigma(0)+E_{d}\right)}{(2 \pi)^{2}} \int_{-\infty}^{\infty} d z G_{0}^{2}(z) \\
& \times \int_{-\infty}^{\infty} d \omega G_{0}(\omega) G_{0}(\omega-z)
\end{aligned}
$$

where we used the relation

$G_{0}^{2}(z)=-\frac{\partial G_{0}(z)}{\partial z}-\frac{2}{\Delta} \delta(z)$

From (21) we obtain:

$\Sigma^{(2)}=-\left(\frac{\pi^{2}}{4}-2\right) \Delta u^{2} \frac{\Sigma(0)+E_{d}}{\Delta}$ 
Taking the lowest order for $\Sigma(0)=0$, we get the equation

$\Sigma^{(2)}(0)=-u^{2} \Delta\left(\frac{\pi^{2}}{4}-2\right) \frac{E_{d}}{\Delta}$

where $u=U /(\pi \Delta)$, and the Green function (2) becomes:

$G_{\sigma}(\omega)=\frac{1}{\omega-E_{d}\left[1+u^{2}\left(\frac{\pi^{2}}{4}-2\right)\right]+i \Delta \operatorname{sgn} \omega}$

a result which is in agreement with $[5,6]$.

\section{Local Density of States}

The local density of states (LDS) for the $d$-electrons is given by the general equation

$\rho(\omega)=-\frac{1}{\pi} \operatorname{Im} G_{\sigma}(\omega)$

For the strong Coulomb repulsion $U$ we obtain from (15) the form:

$\rho(\omega)=\frac{1}{\pi} \frac{\Delta}{\left(\omega-E_{d}^{*}\right)^{2}+\Delta^{2}}$

which contains the Coulomb interaction dependence on $E_{d}^{*}$ and the conductance at zero temperature $G(T=0)$, which is proportional to $\rho(0)$ scales with $1 /\left(\ln ^{2} U / \Delta\right)$.

For small $U /(\pi \Delta)$, the density of states is:

$\rho(\omega)=\frac{1}{\pi} \frac{\Delta}{\left[\omega-\bar{E}_{d}\right]^{2}+\Delta^{2}}$

where $\bar{E}_{d}=E_{d}\left[1+u^{2}\left(\frac{\pi^{2}}{4}-2\right)\right]$ and in this case the conductance decreases with the increasing of the Coulomb interaction as $G(T=0) \sim 1 / u^{2}$.

\section{Magnetic Susceptibility}

The magnetic susceptibility $\chi$ is defined by

$\chi=\frac{g \mu_{B}}{2 H}\left(n_{\uparrow}-n_{\downarrow}\right)$

where $n_{\sigma}$ is given by

$n_{\sigma}=\frac{1}{2}-\frac{1}{\pi} \arctan \left(y_{\sigma}-\sigma h\right)$

where $\sigma= \pm 1, h=g \mu_{B} H / 2 \Delta$, and $y_{\sigma}=\left(\Sigma_{\sigma}+E_{d}\right) / \Delta$. In the weak magnetic field the magnetic susceptibility is given by

$\chi=\frac{\left(g \mu_{B}\right)^{2}}{2} N(0) \bar{\chi}$ where $N(0)=\left[\pi \Delta\left(1+y^{2}\right)\right]$ is the density of states of $d$-electrons and $\bar{\chi}=1+y_{1}$ is the change of the self-energy due to the magnetic field, and we will take the liner term in $h$.

In order to calculate this correction, we consider (9) in presence of magnetic field, as

$y_{\sigma}=-\operatorname{Im} \int_{-\infty}^{0} d x \frac{1}{\left[\frac{1}{u}+F(x, y)\right]\left[x-\left(y_{-\sigma}-\sigma h\right)+i\right]}$

where $F(x, y)$ does not depend on $h$ in the first order. From this equation we obtain, taking $y_{\sigma}=y-\sigma h y_{1}$,

$y_{1}=\frac{\lambda}{1-\lambda}$

where

$\lambda=\operatorname{Im} \int_{-\infty}^{0} d x \frac{1}{\left[\frac{1}{u}+F(x, y)\right](x-y+i)^{2}}$

Using this result in (30), we obtain:

$\chi=\frac{\left(g \mu_{B}\right)^{2}}{2} N(0) \bar{\chi}=\frac{\chi_{K}}{\left[\left(1+y^{2}\right)(1-\lambda)\right]}$

where $\chi_{K}=\left(g \mu_{B}\right)^{2} / 2 \pi \Delta$ and

$\bar{\chi}=\frac{1}{1-\lambda}$

The enhancement parameter $\bar{\chi}$ depends on $u$ as

$\bar{\chi}=1+3 n$

for $u \gg 1$, and

$\bar{\chi}=1+u+\left(3-\frac{\pi^{2}}{4}\right) u^{2}$

for $u \ll 1$.

In the case $u \ll 1$, we calculate

$\chi=\bar{\chi} \frac{1+u+a u^{2}}{1+\left(\frac{\pi u}{2}\right)^{2}\left[1-u+a u^{2}\right]}$

which has a maximum around $u=1 / 2$ due to the competition due to the increase of $\bar{\chi}$ and the decrease of the density of states as a function of $u$.

The result given by (30) can be obtained also using the Hewson renormalized [11] perturbation calculations starting with $\Sigma_{\sigma}(\omega)$ :

$\Sigma_{\sigma}(\omega+\sigma h)=\Sigma_{\sigma}(0)+(\omega+\sigma h) \Sigma_{\sigma}^{\prime}(0)$

The lowest order contribution in the self-energy is

$\Sigma_{\sigma}^{(1)}(h)=U n_{d,-\sigma}(h)$ 
with

$n_{d,-\sigma}=\frac{1}{2}-\frac{1}{\pi} \arctan \left(\frac{E_{d}-\sigma h+\Sigma_{\sigma}(0, h)}{\Delta}\right)$

which gives

$\chi=\frac{\left(g \mu_{B}\right)^{2}}{2} N(0)\left[1-\frac{\partial \Sigma(h)}{\partial h}\right]$

and using now the relation $\Sigma(0, h) \simeq \Sigma^{1}(h)$ we obtain the result given by (30).

\section{Discussion}

We studied the influence of the Coulomb interaction effect in the Anderson model using the method given by Schrieffer and Mattis [10] for the study of the correlation effect on the occurrence of the localized magnetic moments in the Anderson model. The procedure is valid in the low density approximation $(n \ll 1)$ where the particle-particle $t$-matrix dominates the summation to each order in the Coulomb interaction.

In the approximation of the strong Coulomb interaction $t(0) \simeq\left(\pi E_{d}^{*}\right) /\left[\arctan \left(E_{d}^{*}\right) / \Delta\right]$ and in the limit $U \longrightarrow \infty$, it remains finite. The self-energy calculated in this limit is proportional to $(\Delta / \pi) \ln (U / \Delta)$ in agreement with the Haldane [9] scaling result.

The weak coupling limit $(u \ll 1)$ has been performed neglecting the energy dependence of the self-energy, which was approximated by its value at the Fermi energy. The ladder approximation gives a correct result only up to $U^{2}$, but for applications this approximation is relevant.

The results have been applied for the calculation of the density of states in the strong and weak limits and we obtained a Lorenzian form but with an $d$-electron energy containing the influence of the Coulomb interaction. In the case of weak Coulomb interaction $(u \ll 1)$ we showed that the susceptibility presents a maximum at $u=1 / 2$ which is given by the Coulomb interaction effect in the enhancement parameter $\bar{\chi}$ and the density of states.

The simple correction of the self-energy leads to the susceptibility dependence which is in agreement with the expression obtained by Hewson $[11,12]$. These have been obtained neglecting the disorder and fluctuation effects, which together with the Coulomb interaction describe the tunneling in the metallic grains. Such an approximation is valid because a QD is in fact one metallic grain between two leads at low temperatures, and in this case the conduction is strongly affected by the Coulomb interaction.

Acknowledgement We would like to acknowledge the financial support from the Romanian National Research Program PN II-ID-502.

Open Access This article is distributed under the terms of the Creative Commons Attribution Noncommercial License which permits any noncommercial use, distribution, and reproduction in any medium, provided the original author(s) and source are credited.

\section{References}

1. Hewson, A.C.: The Kondo Problem to Heavy Fermions. Cambridge University Press, Cambridge (1993)

2. Beloborodov, I.S., Lopatin, A.V., Vinokur, V.M., Efetov, K.B.: Rev. Mod. Phys. 79, 469 (2007)

3. Gramespacher, T., Matveev, K.A.: Phys. Rev. Lett. 85, 4582 (2000)

4. Yoshida, K., Yamada, K.: Prog. Theor. Phys. 53, 1286 (1975)

5. Yamada, K.: Prog. Theor. Phys. 54, 316 (1975)

6. Horvatić, B., Zlatić, V.: Phys. Status Solidi B 99, 251 (1980)

7. Wilson, K.G.: Rev. Mod. Phys. 47, 733 (1975)

8. Noziéres, P.: J. Low Temp. Phys. 17, 31 (1974)

9. Haldane, F.D.M.: Phys. Rev. Lett. 40, 416 (1978)

10. Schrieffer, J.R., Mattis, D.C.: Phys. Rev. A 140, 1412 (1965)

11. Hewson, A.C.: Phys. Rev. Lett. 70, 4007 (1963)

12. Hewson, A.C.: J. Phys., Condens. Matter 13, 10011 (2001)

13. Son, D.N., Arboleda, N. Jr., Dimo, W.A., Kasai, H.: Eur. Phys. J. B 57, 27 (2007) 